PROCEEDINGS OF THE AMERICAN MATHEMATICAL SOCIETY

Volume 126, Number 7, July 1998, Pages 2183-2189

S 0002-9939(98)04353-6

\title{
RINGS OF CONTINUOUS FUNCTIONS AND THE BRANCH SET OF A COVERING
}

\author{
M. A. MULERO
}

(Communicated by Franklin D. Tall)

\begin{abstract}
This paper gives a characterization of the branch set of a finite covering $X \rightarrow S$ of a topological space $S$, by means of finite $C(S)$-subalgebras $A$ of $C(X)$ that separate points in $X$ and the module $\Omega_{A / C(S)}$ of its Kähler differentials.
\end{abstract}

\section{INTRODUCTION}

The aim of this paper is to characterize the branch set of a finite covering $X \rightarrow S$ of a topological space $S$ by means of algebraic properties of the induced homomorphism $C(S) \rightarrow C(X)$ between their algebras of real-valued continuous functions.

Our starting point is the well-known result that, in the realm of real-compact spaces, every space $X$ is determined by the algebra $C(X)$ of all real-valued continuous functions defined on it. Moreover, each continuous map $X \rightarrow S$ defines, by composition, a homomorphism of $\mathbb{R}$-algebras $C(S) \rightarrow C(X)$ and, in this way, continuous maps between real-compact spaces are in one-to-one correspondence with homomorphisms between their algebras of continuous functions. From this equivalence it follows that there should be close relationships between the topological properties of spaces and maps and the algebraic properties of the corresponding algebras and homomorphisms. Examples of results showing these relationships are the characterization of local homeomorphisms by means of rings of germs of continuous functions in [7], the correspondence between finite unbranched coverings of a topological space $X$ and separable $C(X)$-algebras which are finitely generated projective $C(X)$-modules [2], the characterization of the dimension of a topological space $X$ in terms of dense subalgebras of $C(X)$ [14], and the going-up and going-down theorems for the homomorphism $C(S) \rightarrow C(X)$ defined by an open and closed map $X \rightarrow S[11]$.

In this paper we are concerned with finite (branched) coverings. By a finite covering we shall mean an open and closed continuous map $\pi: X \rightarrow S$ with finite fibres and such that any pair of points in the same fibre have disjoint neighbourhoods in $X$. Note that a finite covering $\pi: X \rightarrow S$ is not necessarily a local homeomorphism (for example, $\pi: \mathbb{C} \rightarrow \mathbb{C}, \pi(z)=z^{2}$ ). The set of points in $X$ at which $\pi$ is not a local homeomorphism is called the branch set of $\pi$.

Received by the editors January 30, 1996 and, in revised form, January 1, 1997.

1991 Mathematics Subject Classification. Primary 54C40, 13B10, 54C10.

Key words and phrases. Rings of continuous functions, branched covering, Kähler differentials.

(C)1998 American Mathematical Society 
In [12] it is proved that a continuous map between topological manifolds $\pi$ : $X \rightarrow S$ is a finite covering if and only if the induced homomorphism $C(S) \rightarrow C(X)$ is integral and flat. The aim of the present paper is to complete this result by providing a characterization of the branch set in terms of the rings of continuous functions.

We must bear in mind that in Algebraic Geometry, the points of the branch set of a covering $X \rightarrow S$ of an algebraic variety $S$ are just the points where the germ of the module $\Omega_{A(X) / A(S)}$ of Kähler differentials of the algebra $A(X)$ of algebraic functions on $X$ over the corresponding algebra $A(S)$ is not zero. In Differential Geometry, there is an entirely analogous result for differentiable manifolds (essentially, it is a translation of the Inverse Function Theorem in terms of differentials).

One may wonder if the above result for algebraic and differentiable functions also holds in the topological case. If you simply copy the statement, it is not true; we give an example of a finite branched covering $X \rightarrow S$ such that $\Omega_{C(X) / C(S)}=0$. But the natural way to translate results from algebra to topology is to replace rings of algebraic functions by dense subalgebras of continuous functions, as was done in [14] to characterize the dimension.

In accordance with this general principle, we obtain the following result:

Theorem. Let $\pi: X \rightarrow S$ be a finite covering and let $A$ be a finite $C(S)$-subalgebra of $C(X)$ separating points in $X$. Then, $\pi$ is a local homeomorphism at a point $x \in X$ if and only if $\left(\Omega_{A / C(S)}\right)_{x}=0$.

This theorem is not applicable to the whole algebra $C(X)$, because $C(X)$ is not a finite $C(S)$-algebra whenever the branch set of $\pi: X \rightarrow S$ is not empty (see [12]).

When the base space $S$ is a metric space of finite dimension, then we shall also prove the existence of subalgebras of $C(X)$ satisfying the conditions of the above theorem.

\section{PRELIMINARIES}

Concerning rings of continuous functions, we shall use the same notation and terminology as in [5].

Every continuous map $\pi: X \rightarrow S$ defines, by composition, a homomorphism of rings $C(S) \rightarrow C(X)$. This homomorphism defines in $C(X)$ a natural algebra structure over $C(S)$. Given $g \in C(S)$, if no confusion is possible, the function $g \circ \pi$ will also be denoted by $g$.

The set of all prime ideals in $C(X)$, i.e., the prime spectrum, will be denoted by Spec $C(X)$. Each point $x \in X$ defines a maximal ideal $\mathfrak{m}_{x}=\{f \in C(X): f(x)=$ $0\}$. If $M$ is a $C(X)$-module and $\mathfrak{p}$ is a prime ideal in $C(X)$, we shall denote by $M_{\mathfrak{p}}$ the localization, or module of fractions, of $M$ with respect to the multiplicatively closed subset $C(X)-\mathfrak{p}$, and we shall write $M_{x}$ instead of $M_{\mathfrak{m}_{x}}$. The same notation will be used for modules over subalgebras $A$ of $C(X)$. The definition and basic properties of rings and modules of fractions may be found in [1] and [9].

The zero-set of a function $f$ is the set $\mathrm{Z}(f)=\{x \in X: f(x)=0\}$ and the cozero-set of $f$ is $\operatorname{coz}(f)=X-\mathrm{Z}(f)$.

An ideal $I$ of $C(X)$ is said to be a z-ideal if $\mathrm{Z}(f)=\mathrm{Z}(g)$ and $f \in I$ imply $g \in I$. This condition is equivalent to the following one: $\mathrm{Z}(f) \subseteq \mathrm{Z}(g)$ and $f \in I$ imply $g \in I$, because $\mathrm{Z}(f) \cap \mathrm{Z}(g)=\mathrm{Z}\left(f^{2}+g^{2}\right)$.

We recall the definitions of derivations and differentials and for properties we refer to [3] or [9]. 
Definitions 0.1. Let $k$ be a ring. Let $A$ be a $k$-algebra and let $M$ be an $A$ module. A $k$-derivation from $A$ to $M$ is a $k$-linear map $D: A \rightarrow M$ that satisfies $D(a \cdot b)=a \cdot D(b)+b \cdot D(a)$. (Observe that this condition implies $D(\alpha)=0$ for every $\alpha \in k$.) The set of all $k$-derivations from $A$ to $M$ is written $\operatorname{Der}_{k}(A, M)$, and it becomes an $A$-module in a natural way. Since the composition of a $k$-derivation $D: A \rightarrow M$ with a homomorphism of $A$-modules $f: M \rightarrow M^{\prime}$ is a $k$-derivation $f \circ D: A \rightarrow M^{\prime}$, we have a covariant functor $M \rightarrow \operatorname{Der}_{k}(A, M)$ from the category of $A$-modules to itself. This is a representable functor, i.e., there exists an $A$-module $\Omega_{A / k}$ endowed with a derivation $d: A \rightarrow \Omega_{A / k}$ satisfying a universal property

$$
\operatorname{Der}_{k}(A, M)=\operatorname{Hom}_{A}\left(\Omega_{A / k}, M\right) .
$$

The $A$-module $\Omega_{A / k}$ is called the $A$-module of Kähler differentials of $A$ over $k$.

Explicitly, $\Omega_{A / k}$ can be taken to be the module $\Delta / \Delta^{2}$, where $\Delta$ is the kernel of the homomorphism $m: A \otimes_{k} A \rightarrow A, m(a \otimes b)=a \cdot b$. Then, the derivation $d: A \rightarrow \Omega_{A / k}$ is defined to be $d a=$ residue class of $(a \otimes 1-1 \otimes a)$ in $\Delta / \Delta^{2}$. It is clear that $d \alpha=0, \forall \alpha \in k$.

These concepts of derivations and differentials play a major role in Algebraic and Differential Geometry (see [3, Chapter 16]). For example, if $X$ is a $C^{\infty}$-manifold, the $C^{\infty}(X)$-module of derivations $\operatorname{Der}_{\mathbb{R}}\left(C^{\infty}(X), C^{\infty}(X)\right)$ is just the module of tangent vector fields and the tangent space at a point $x \in X$ is the vector space $\mathrm{T}_{x} X=\operatorname{Der}_{\mathbb{R}}\left(C^{\infty}(X)_{x}, \mathbb{R}\right)$. In the topological case, this concept of tangent space has no meaning, since it is not difficult to prove that $\operatorname{Der}_{\mathbb{R}}\left(C(X)_{x}, \mathbb{R}\right)=0$ and $\operatorname{Der}_{\mathbb{R}}(C(X), C(X))=0$. However, as proved in [6], the module $\Omega_{C(X) / \mathbb{R}}$ is, in general, different from zero.

\section{Characterization OF THE BRANCH SET}

Definitions 1.1. A map $\pi: X \rightarrow S$ is said to be a trivial covering of $S$ if the space $X$ has a cover by pairwise disjoint open sets each of them homeomorphic to $S$ by $\pi$.

A map $\pi: X \rightarrow S$ is said to be an unbranched covering of $S$ if every point of $S$ has a neighbourhood $U$ such that $\pi: \pi^{-1}(U) \rightarrow U$ is a trivial covering.

By a finite covering of $S$ we shall mean an open and closed continuous map $\pi: X \rightarrow S$ with finite fibres and such that any pair of points in the same fibre have disjoint neighbourhoods in $X$.

Since the image of a finite covering is an open and closed subset of $S$, for our purposes there is no loss of generality in assuming that every finite covering is surjective.

The branch set of a finite covering $\pi$ is the closed set $B_{\pi}$ of all points in $X$ where $\pi$ is not a local homeomorphism. The closed set $\pi\left(B_{\pi}\right)$ will be denoted by $R_{\pi}$ and its complement $S-R_{\pi}$ will be denoted by $S_{0}$.

We shall use some easy-to-prove properties of finite coverings.

Properties 1.2. Let $\pi: X \rightarrow S$ be a finite covering.

(i) If $s \in S$ and $\pi^{-1}(s)=\left\{x_{1}, \ldots, x_{n}\right\}$, then there exist neighbourhoods $U$ of $s$ satisfying

$$
\pi^{-1}(U)=U_{1} \sqcup \cdots \sqcup U_{n}
$$

where $U_{i}$ is a neighbourhood of $x_{i}$ such that

$$
\pi\left(U_{i}\right)=U \text { for every } i \text {, and } U_{i} \cap U_{j}=\emptyset \text { if } i \neq j .
$$


(ii) A point $s$ belongs to $S_{0}$ if and only if the cardinality of the fibres of $\pi$ is constant on some neighbourhood of $s$, or equivalently, if the covering $\pi$ is trivial on some neighbourhood of $s$.

(iii) Let $X_{0}=\pi^{-1}\left(S_{0}\right)$. The restriction $\pi: X_{0} \rightarrow S_{0}$ is an unbranched covering and it is said to be the unbranched part of $\pi$.

Definitions 1.3. Let $k$ be a ring and let $A$ be a $k$-algebra. $A$ is said to be a finite $k$-algebra if it is finitely generated as a $k$-module, i.e., if there exists a finite set of elements $a_{1}, \ldots, a_{n} \in A$ such that every element of $A$ is a linear combination of $a_{1}, \ldots, a_{n}$ with coefficients in $k$, i.e., $A=k a_{1}+\cdots+k a_{n} . A$ is a finitely-generated $k$ algebra if there exists a finite set of elements $a_{1}, \ldots, a_{n} \in A$ such that every element of $A$ is a polynomial in $a_{1}, \ldots, a_{n}$ with coefficients in $k$, i.e., $A=k\left[a_{1}, \ldots, a_{n}\right]$.

Definitions 1.4. Let $Y$ be a subset of a topological space $X$ and let $A$ be an $\mathbb{R}$ subalgebra of $C(X)$. We shall say that $A$ separates points in $Y$ if for any two points $x, y \in Y$ there exists $f \in A$ such that $f(x) \neq f(y)$.

Lemma 1.5. Let $\pi: X \rightarrow S$ be a closed map with finite fibres. If $s$ is a point in $S$ and $\pi^{-1}(s)=\left\{x_{1}, \ldots, x_{n}\right\}$, then:

(i) $C(X)_{s}$ is naturally isomorphic to the direct product of the rings of germs at the points $x_{i}$ :

$$
C(X)_{s}=C(X)_{x_{1}} \times \cdots \times C(X)_{x_{n}} .
$$

(ii) If $A$ is a finite $C(S)$-subalgebra of $C(X)$ that separates points in $X$, then $A_{s}$ is naturally isomorphic to the direct product of the localizations $A_{x_{i}}$ of $A$ in the maximal ideals $\mathfrak{m}_{x_{i}} \cap A$ :

$$
A_{s}=A_{x_{1}} \times \cdots \times A_{x_{n}} .
$$

Proof. (i) To prove that the natural homomorphism $C(X)_{s} \rightarrow C(X)_{x_{1}} \times \cdots \times$ $C(X)_{x_{n}}$ is an isomorphism, it is enough to prove that, for every maximal ideal $\mathfrak{m}$ in $C(X)$, the homomorphism $\left(C(X)_{s}\right)_{\mathfrak{m}} \rightarrow\left(C(X)_{x_{1}}\right)_{\mathfrak{m}} \times \cdots \times\left(C(X)_{x_{n}}\right)_{\mathfrak{m}}$ is an isomorphism [1, Proposition 3.9].

Recall that if $\mathfrak{p}$ is a prime ideal in $C(X)$, then $\operatorname{Spec}\left(C(X)_{\mathfrak{p}}\right)$ is naturally homeomorphic to the subspace of $\operatorname{Spec} C(X)$ of prime ideals contained in $\mathfrak{p}[9, \S 4$, Example 2]. The prime ideals in $C(X)$ containing a given prime ideal form a chain $[5,14.3$.(c)]. Therefore, if a prime ideal $\mathfrak{p}$ is not contained in a maximal ideal $\mathfrak{m}$, there are no prime ideals in $C(X)$ contained in both $\mathfrak{p}$ and $\mathfrak{m}$. These prime ideals simultaneously contained in $\mathfrak{p}$ and $\mathfrak{m}$ are just the prime ideals in the ring $\left(C(X)_{\mathfrak{p}}\right)_{\mathfrak{m}}$, so that $\left(C(X)_{\mathfrak{p}}\right)_{\mathfrak{m}}=0$.

From this it follows that if $\mathfrak{m}=\mathfrak{m}_{x_{i}}$, the above homomorphism is an isomorphism:

$$
\left(C(X)_{s}\right)_{x_{i}}=C(X)_{x_{i}} \rightarrow 0 \times \cdots \times C(X)_{x_{i}} \times \cdots \times 0=C(X)_{x_{i}} .
$$

Let $\mathfrak{m} \neq \mathfrak{m}_{x_{i}}(\forall i=1, \ldots, n)$. Then, there exists $f \in \mathfrak{m}$ such that $f\left(x_{i}\right) \neq 0(\forall i=$ $1, \ldots, n)$. Since $\pi(\mathrm{Z}(f))$ is a closed subset in $S$ and $s \notin \pi(\mathrm{Z}(f))$, there exists a function $g \in C(S)$ such that $\pi(\mathrm{Z}(f)) \subseteq \mathrm{Z}(g)$ and $g(s) \neq 0$. Since the maximal ideal $\mathfrak{m}$ is a $z$-ideal $[5,2.7]$ and $\mathrm{Z}(f) \subseteq \mathrm{Z}(g \circ \pi)$, one has that $g \circ \pi$ belongs to $\mathfrak{m}$, but $g \circ \pi \notin \mathfrak{m}_{s}$. Hence, the prime ideal $\mathfrak{p}=\mathfrak{m} \cap C(S)$ is not contained in $\mathfrak{m}_{s}$, so that $\left(C(X)_{s}\right)_{\mathfrak{m}}=\left(\left(C(X)_{s}\right)_{\mathfrak{p}}\right)_{\mathfrak{m}}=0$ and $\left(C(X)_{x_{1}}\right)_{\mathfrak{m}} \times \cdots \times\left(C(X)_{x_{n}}\right)_{\mathfrak{m}}=0$.

(ii) The local ring $C(S)_{s}$ is a Henselian ring [13, Chap. VII, Prop. 4], i.e., every finite $C(S)_{s}$-algebra is a direct product of local algebras. If $A$ is a finite $C(S)$ subalgebra of $C(X)$, then $A_{s}$ is a finite $C(S)_{s}$-algebra and so the local algebras $A_{x_{i}}$ 
are factors of $A_{s}$. Since $A_{s}$ is a subalgebra of $C(X)_{s}=C(X)_{x_{1}} \times \cdots \times C(X)_{x_{n}}$, these $A_{x_{i}}$ are the only factors of $A_{s}$, i.e., $A_{s}=A_{x_{1}} \times \cdots \times A_{x_{n}}$.

Theorem 1.6. Let $\pi: X \rightarrow S$ be a finite covering. If $A$ is a finite $C(S)$-subalgebra of $C(X)$ that separates points in $X$, then

(i) $B_{\pi}=\left\{x \in X:\left(\Omega_{A / C(S)}\right)_{x} \neq 0\right\}$,

(ii) $R_{\pi}=\left\{s \in S:\left(\Omega_{A / C(S)}\right)_{s} \neq 0\right\}$.

Proof. (i) Let $x$ be a point in $X$ and $s=\pi(x)$. If $\pi$ is a homeomorphism in some neighbourhood of $x$, i.e., if $x \notin B_{\pi}$, then $C(S)_{s}=A_{x}=C(X)_{x}$ and since the module of differentials commutes with base change and with localization [3, Propositions 16.4 and 16.9], we have

$$
\left(\Omega_{A / C(S)}\right)_{x}=\left(\left(\Omega_{A / C(S)}\right)_{s}\right)_{x}=\left(\Omega_{A_{s} / C(S)_{s}}\right)_{x}=\Omega_{A_{x} / C(S)_{s}}=0 .
$$

Conversely, if $\left(\Omega_{A / C(S)}\right)_{x}=0$, then, changing the base ring [3, Proposition 16.4], we have

$$
\left(\Omega_{A / C(S)}\right)_{x} \otimes_{C(S)} C(S) / \mathfrak{m}_{s}=\Omega_{\bar{A} / \mathbb{R}}=0,
$$

where $\bar{A}=A_{x} / \mathfrak{m}_{s} A_{x}$. This implies that $\mathbb{R}=C(S) / \mathfrak{m}_{s} \rightarrow \bar{A}=A_{x} / \mathfrak{m}_{s} A_{x}$ is a separable $\mathbb{R}$-algebra [3, Proposition 16.10] and therefore $\bar{A}$ has no nilpotent elements. Since $\bar{A}$ is finitely generated as a vector space over $\mathbb{R}$, every prime ideal in $\bar{A}$ is a maximal ideal [1, Corollary 5.8]. Thus, the only prime ideal in the local algebra $\bar{A}$ is the maximal ideal $\overline{\mathfrak{m}}=\left(\mathfrak{m}_{x} \cap A\right) \bar{A}$. Since $\bar{A}$ has no nilpotent elements, $\overline{\mathfrak{m}}=0$. Hence, $\bar{A}=\bar{A} / \overline{\mathfrak{m}}=\mathbb{R}$. It follows, by Nakayama's Lemma [1, Proposition 2.8], that the homomorphism $C(S)_{s} \rightarrow A_{x}$ is surjective and therefore, for any $f \in A$ there exists $g \in C(S)$ such that $f=g$ on some neighbourhood of $x$. Since $A$ is finitely generated as a $C(S)$-module, we conclude that there is a neighbourhood $U$ of $x$ where any $f \in A$ coincides with some $g \in C(S)$. Since $A$ separates points in $X$, the restriction of $\pi$ to this neighbourhood $U$ is one-to-one, and consequently $\pi$ is a homeomorphism between $U$ and $\pi(U)$.

(ii) This is a consequence of (i): If $s \in S$ and $\pi^{-1}(s)=\left\{x_{1}, \ldots, x_{n}\right\}$, then, by Lemma 1.5, $A_{s}=A_{x_{1}} \times \cdots \times A_{x_{n}}$ and therefore [3, Corollary 16.16] $\Omega_{A_{s} / C(S)_{s}}=$ $\bigoplus \Omega_{A_{x_{i}} / C(S)_{s}}$. From this it follows that $\Omega_{A_{s} / C(S)_{s}}$ is not the zero module if and only if some $x_{i}$ is in $B_{\pi}$, i.e., if and only if $s \in R_{\pi}$.

The following example shows that it is not possible to characterize the branch sets $B_{\pi}, R_{\pi}$, by means of the module of relative Kähler differentials $\Omega_{C(X) / C(S)}$. This is not an exceptional example, since we have proved in [10] that, when the supremum of the cardinalities of the fibres of a covering $\pi: X \rightarrow S$ is finite, then $\Omega_{C(X) / C(S)}=0$. The proof of the statement for the case given in the example is due to J. B. Sancho de Salas.

Example 1.7. Let $X=\left\{(x, y) \in \mathbb{R}^{2}: x^{2}+y^{2}=1\right\}$, and define $\pi: X \rightarrow S=$ $[-1,1]$ by $\pi(x, y)=x$. Clearly, $\pi$ is a finite covering and the branch set $B_{\pi}$ has two points: $(-1,0)$ and $(1,0)$. We are going to prove that $\Omega_{C(X) / C(S)}=0$. Since the $C(X)$-module $\Omega_{C(X) / C(S)}$ is generated by $\{d f: f \in C(X)\}$, it is enough to prove that $d f=0$, for every $f \in C(X)$.

Let $\sigma: X \rightarrow X$ be the homeomorphism $\sigma(x, y)=(x,-y)$. Clearly $\pi \circ \sigma=\pi$ and, given $f \in C(X)$ one has that $f=f \circ \sigma$ if and only if $f \in C(S)$, i.e., if $f=g \circ \pi$, for some $g \in C(S)$. Every $f \in C(X)$ may be written as $f=\frac{1}{2}(f+f \circ \sigma)+\frac{1}{2}(f-f \circ \sigma)$ and $d f=\frac{1}{2} d(f-f \circ \sigma)$, because $f+f \circ \sigma=(f+f \circ \sigma) \circ \sigma$ and consequently 
$d(f+f \circ \sigma)=0$ in $\Omega_{C(X) / C(S)}$. Thus, we have only to prove that $d f=0$ when $f=-f \circ \sigma$. If $f=-f \circ \sigma$, then $f$ has a continuous cubic root $h$, i.e., $f=h^{3}$; and, clearly, $h=-h \circ \sigma$. Since $h^{2}=h^{2} \circ \sigma$, one has that $0=d h^{2}=2 h d h$ and, consequently, $d f=d h^{3}=h^{2} d h=0$.

To complete this example, we take a finite $C(S)$-subalgebra $A$ of $C(X)$ separating points in $X$ and we check that the module $\Omega_{A / C(S)}$ gives the branch set.

Denote by $x$ and $y$ respectively, the first and the second projection map on $X$ and let $A=C(S)[y]$. Clearly, $A$ separates points in $X$ and it is not difficult to prove that $A$ is isomorphic to the following quotient of the ring of polynomials $A=C(S)[t] /\left(t^{2}+x^{2}-1\right)$, so that $A$ is a finite $C(S)$-algebra. The module of differentials $\Omega_{A / C(S)}$ is the quotient of the free $A$-module of rank one with base $d y$ by the submodule generated by $d\left(y^{2}+x^{2}-1\right)=2 y d y$ (see $\left.[3, \S 16.1]\right)$. That is to say,

$$
\Omega_{A / C(S)}=A d y / 2 y d y \simeq A /(y)=C(S)[t] /\left(t^{2}+x^{2}-1, t\right)=C(S) /\left(x^{2}-1\right) .
$$

The germ of the $A$-module $\Omega_{A / C(S)}=A /(y)$ is different from zero exactly at the points of $X$ where the function $y$ is not locally invertible in $A$, i.e., in $(-1,0)$ and $(1,0)$. And, as a $C(S)$-module, the germ of $\Omega_{A / C(S)}=C(S) /\left(x^{2}-1\right)$ is different from zero exactly at the points of $S$ where the function $x^{2}-1$ is not locally invertible in $C(S)$, i.e., in $x=-1$ and $x=1$.

\section{Finite Subalgebras}

Let $\pi: X \rightarrow S$ be a finite covering of $S$. Then in this section we shall be concerned with the existence of subalgebras of $C(X)$ satisfying the conditions of Theorem 1.6, i.e., finite $C(S)$-subalgebras that separate points in $X$.

Lemma 2.1. Let $\pi: X \rightarrow S$ be a finite covering of a metric space $S$ of finite cover dimension. If the supremum of the cardinalities of the fibres of $\pi$ is finite, then there exist finitely-generated $C(S)$-subalgebras of $C(X)$ that separate points in $X$.

Proof. Let $\pi: X_{0} \rightarrow S_{0}$ be the unbranched part of $\pi: X \rightarrow S$. If the cozero-set $U=\operatorname{coz}(g)$ of a function $g$ in $C(S)$ is contained in $S_{0}$ and $\pi: \pi^{-1}(U) \rightarrow U$ is a trivial covering, i.e., $\pi^{-1}(U)=U_{1} \sqcup \cdots \sqcup U_{m}$ and each $U_{i}$ is homeomorphic to $U$ by $\pi$, then the functions $f_{i}$ defined on $X$ by $f_{i}=g$ on $U_{i}$ and $f_{i}=0$ on $X-U_{i}$ are trivially continuous on $X$. Clearly, $C(S)\left[f_{1}, \ldots, f_{m}\right]$ separates points in $\pi^{-1}(U)$.

It is not difficult to see that, since $S_{0}$ is a metric space of finite cover dimension, every open cover of $S_{0}$ has a refinement that may be written as the union of finitely many families consisting of pairwise disjoint open sets; therefore the unbranched covering $\pi: X_{0} \rightarrow S_{0}$ becomes trivial on some finite open cover of $S_{0}$. Moreover, the members of this finite cover are cozero-sets in $S$, since $S$ is a metric space.

Now, it is clear that $C(X)$ has a finitely-generated subalgebra $C(S)\left[f_{1}, \ldots, f_{k}\right]$ that separates points in $X_{0}$.

Consider the finite covering $\pi: \pi^{-1}\left(R_{\pi}\right) \rightarrow R_{\pi}$ and let $\pi: \pi^{-1}\left(\left(R_{\pi}\right)_{0}\right) \rightarrow$ $\left(R_{\pi}\right)_{0}$ be its unbranched part. As proved above, there is a finite family of functions $f_{1}^{\prime}, \ldots, f_{l}^{\prime}$ in $C\left(\pi^{-1}\left(R_{\pi}\right)\right)$ such that $C\left(\pi^{-1}\left(R_{\pi}\right)\right)\left[f_{1}^{\prime}, \ldots, f_{l}^{\prime}\right]$ separates points in $\pi^{-1}\left(\left(R_{\pi}\right)_{0}\right)$. These functions may be continuously extended to the whole space $X$, since $X$ is the inverse image by a proper map of the paracompact space $S$ and it is therefore a paracompact [4, Theorem 5.1.35] and a fortiori, normal space. The subalgebra $C(S)\left[f_{1}, \ldots, f_{k}, f_{1}^{\prime}, \ldots, f_{l}^{\prime}\right]$ separates points in $X_{0} \cup \pi^{-1}\left(\left(R_{\pi}\right)_{0}\right)$. In this way, in a finite number of steps, we obtain a finitely-generated $C(S)$-subalgebra of 
$C(X)$ that separates points in $X$ since, if the supremum of the cardinalities of the fibres of a covering $\pi: X \rightarrow S$ is equal to $n$, then the cardinality of the fibres of $\pi: \pi^{-1}\left(R_{\pi}\right) \rightarrow R_{\pi}$ is bounded by $n-1$.

Corollary 2.2. Let $X$ and $S$ be connected topological manifolds (Hausdorff and paracompact) of finite dimension and let $\pi: X \rightarrow S$ be a finite covering. Then, there are finite $C(S)$-subalgebras in $C(X)$ that separate points in $X$.

Proof. If $X$ and $S$ are topological manifolds, then the supremum of the cardinalities of the fibres of a finite covering $\pi: X \rightarrow S$ is finite [8, Theorem 3.7] so that, by 2.1, $C(X)$ has finitely-generated $C(S)$-subalgebras that separate points in $X$. These finitely-generated subalgebras are finite $C(S)$-algebras because, as proved in [12], the homomorphism $C(S) \rightarrow C(X)$ is integral.

\section{ACKNOWLEDGMENT}

This paper is based on part of the author's doctoral dissertation, written under supervision of Professor J. B. Sancho de Salas. The author wishes to express her gratitude to him and to Professor J. A. Navarro González for the valuable advice and encouragement given during the preparation of this paper.

\section{REFERENCES}

[1] M. Atiyah and I. G. Mac-Donald, Introduction to Commutative Algebra, Addison-Wesley, 1969. MR 39:4129

[2] L. Childs, On covering spaces and Galois extensions, Pacific J. Math. 37 (1971), 29-33. MR 46:2630

[3] D. Eisenbud, Commutative Algebra with a View Toward Algebraic Geometry, SpringerVerlag, 1995. MR 97a:13001

[4] R. Engelking, General Topology, Hermann Verlag, 1989. MR 91c:54001

[5] L. Gillman and M. Jerison, Rings of Continuous Functions, Springer Verlag, 1976. MR 53:11352

[6] F. Gómez, The number of generators of the algebra of Kähler differentials, Demonstratio Math. Vol XXIII, N 2 (1990), 375-383. MR 92b:13036

[7] K. R. Goodearl, Local isomorphisms of algebras of continuous functions, Journal of the London Math. Soc. (2) 16 (1977), 348-356. MR 58:2203

[8] L. F. McAuley and E. E. Robinson. Discrete open and closed mappings on generalized continua and Newman's property, Can. J. Math. vol XXXVI, 6 (1984), 1081-1112. MR 86h:54012

[9] H. Matsumura, Commutative Ring Theory, Cambridge University Press, 1986. MR 88h:13001

[10] M. A. Mulero, Revestimientos finitos y álgebras de funciones continuas, Ph. D. Thesis, Universidad de Extremadura, 1992.

[11] M. A. Mulero, Algebraic properties of rings of continuous functions, Fund. Math., 149 (1996), $55-66$. MR 97c: 16038

[12] M. A. Mulero, Algebraic characterization of finite (branched) coverings, (Preprint).

[13] M. Raynaud, Anneaux Locaux Henséliens. Lecture Note in Mathematics 169. Springer-Verlag (1970). MR 43:3252

[14] J. B. Sancho and M. T. Sancho, Dimension of dense subalgebras of $C(X)$, Proc. of the A.M.S. Vol.105 (2) (1989). MR 89f:54036

Departamento de Matemáticas, Universidad de Extremadura, 06071 Badajoz, Spain

E-mail address: mamulero@ba.unex.es 